\title{
Identification and validation of quantitative trait loci for kernel traits in common wheat (Triticum aestivum L.)
}

Hong Liu' ${ }^{1 \dagger}$, Xiaotao Zhang ${ }^{1,4+}$, Yunfeng Xu' , Feifei Ma ${ }^{1,4}$, Jinpeng Zhang ${ }^{2}$, Yanwei Cao ${ }^{1,4}$, Lihui $\mathrm{Li}^{2^{*}}$ and Diaoguo $\mathrm{An}^{1,3^{*}}$ (D)

\begin{abstract}
Background: Kernel weight and morphology are important traits affecting cereal yields and quality. Dissecting the genetic basis of thousand kernel weight (TKW) and its related traits is an effective method to improve wheat yield.

Results: In this study, we performed quantitative trait loci (QTL) analysis using recombinant inbred lines derived from the cross 'PuBing3228 × Gao8901' (PG-RIL) to dissect the genetic basis of kernel traits. A total of 17 stable QTLs related to kernel traits were identified, notably, two stable QTLs QTkw.cas-1A.2 and QTkw.cas-4A explained the largest portion of the phenotypic variance for TKW and kernel length $(K L)$, and the other two stable QTLs QTkw.cas6A.1 and QTkW.cas-7D.2 contributed more effects on kernel width (KW). Conditional QTL analysis revealed that the stable QTLs for TKW were mainly affected by KW. The QTLs QTKW.cas-7D.2 and QKW.cas-7D.1 associated with TKW and KW were delimited to the physical interval of approximately $3.82 \mathrm{Mb}$ harboring 47 candidate genes. Among them, the candidate gene TaFT-D1 had a 1 bp insertions/deletion (InDel) within the third exon, which might be the reason for diversity in TKW and KW between the two parents. A Kompetitive Allele-Specific PCR (KASP) marker of TaFT-D1 allele was developed and verified by PG-RIL and a natural population consisted of 141 cultivar/lines. It was found that the favorable TaFT-D1 (G)-allele has been positively selected during Chinese wheat breeding. Thus, these results can be used for further positional cloning and marker-assisted selection in wheat breeding programs.
\end{abstract}

Conclusions: Seventeen stable QTLs related to kernel traits were identified. The stable QTLs for thousand kernel weight were mainly affected by kernel width. TaFT-D1 could be the candidate gene for QTLs QTkw.cas-7D.2 and QKW.cas-7D.1.

Keywords: Kernel traits, Quantitative trait locus, TaFT-D1, KASP marker, Triticum aestivum

\footnotetext{
*Correspondence: lilihui@caas.cn; dgan@sjziam.ac.cn

${ }^{\dagger}$ Hong Liu and Xiaotao Zhang contributed equally to this work.

${ }^{2}$ The National Key Facility for Crop Gene Resources and Genetic Improvement, Institute of Crop Science, Chinese Academy of Agricultural Sciences, Beijing 100081, China

${ }^{1}$ Center for Agricultural Resources Research, Institute of Genetics and

Developmental Biology, Chinese Academy of Sciences, Shijiazhuang 050021,

China

Full list of author information is available at the end of the article
}

(c) The Author(s). 2020 Open Access This article is licensed under a Creative Commons Attribution 4.0 International License, which permits use, sharing, adaptation, distribution and reproduction in any medium or format, as long as you give appropriate credit to the original author(s) and the source, provide a link to the Creative Commons licence, and indicate if changes were made. The images or other third party material in this article are included in the article's Creative Commons licence, unless indicated otherwise in a credit line to the material. If material is not included in the article's Creative Commons licence and your intended use is not permitted by statutory regulation or exceeds the permitted use, you will need to obtain permission directly from the copyright holder. To view a copy of this licence, visit http://creativecommons.org/licenses/by/4.0/ The Creative Commons Public Domain Dedication waiver (http://creativecommons.org/publicdomain/zero/1.0/) applies to the data made available in this article, unless otherwise stated in a credit line to the data. 


\section{Background}

Common wheat (Triticum aestivum L.) is one of the most important cereal crops for feeds $40 \%$ of population in the world (http://www.fao.org/). Wheat yield is determined by thousand kernel weight (TKW), kernel number per spike, and effective tiller number [1]. Among them, TKW is the most stable and highest heritable trait, and it is also an important selection target for the genetic improvement of wheat yield [2]. Kernel weight is a complex yield component, which is mainly affected by kernel length (KL), kernel width (KW), kernel length / kernel width (KL/W) and kernel thickness [3]. Therefore, exploring the genetic variation of TKW and its related traits is an effective approach to increase wheat yield [4].

A large number of genes related to kernel weight and morphological traits have been identified in crop. For instance, in rice, GS3, qGL3, GL4 and GLW7 were associated with kernel weight, GW2, GW5, GS5 and GW8 were associated with kernel width [5-12]. Recently, several genes associated with kernel weight have been identified in wheat through comparative genomics approaches, thereby providing an in-depth understanding of the molecular basis of TKW. For example, TaGW2 and TaDA1, which encode an E3 RING ligase [13-15] and a ubiquitin receptor [16], respectively. Both of them are conserved component of the ubiquitin-proteasome pathway and negatively regulate wheat kernel size. In addition, TaGS5$3 A$ [17] and TaFlo2-A1 [18], which encode a serine carboxypeptidase and a protein containing tetratricopeptide repeat motif, respectively, both can regulate kernel size and weight. Genes involved in starch and sucrose metabolism pathways also affect wheat kernel size, such as the cell wall invertase TaCwi-A1 [19], the sucrose synthases TaSus1 and TaSus2 [20], ADP-glucose pyrophosphorylase TaAGP-S1-7A and TaAGP-L-1B [21].

Previous researches have shown that conditional QTL mapping has been used to study genetic basis of complex traits in crops [22, 23]. In wheat, conditional QTL analysis were carried out to evaluate the static genetic control of traits at different growth stages for kernel size and weight $[23,24]$ and yield [25]; to reveal the dynamic genetic factors of plant height $[26,27]$; and to reveal the genetic contribution of different nitrogen and phosphorus supplement environments factors to QTL expression by dissecting QTLs based on trait values conditioned [28].

Recently, high-density single nucleotide polymorphism (SNP) arrays technology provides a superior approach to identify QTLs for wheat kernel-related traits [29-31]. To date, numerous QTLs for kernel traits have been identified on almost 21 wheat chromosomes [32-35]. Remarkably, major stable QTLs distributed on chromosomes 1A, 1B, $2 \mathrm{D}, 3 \mathrm{D}, 4 \mathrm{~A}, 4 \mathrm{~B}, 5 \mathrm{~A}, 7 \mathrm{D}$ can be identified in recombinant inbred line (RIL) populations with different genetic backgrounds [36-40]. Moreover, several yield-related QTLs have been fine mapped and cloned, for example, the major QTL affecting kernel number and kernel weight on chromosome 2AL (GNI-A1) in tetraploid wheat [41, 42]. However, most QTLs associated with kernel traits were mapped by a low-density genetic linkage map with large confidence interval. Only a few QTLs flanking markers were converted into Kompetitive Allele Specific PCR (KASP) markers that can be used in molecular breeding.

Using a RIL population derived from 'PuBing 3228 $(\mathrm{P} 3228) \times$ Gao8901 (G8901)', the objectives of this study were to (i) identify stable and major QTLs for TKW, KL, $\mathrm{KW}$ and KL/W under different field conditions; (ii) reveal the contribution of the other kernel traits to TKW using conditional QTL analysis; (iii) predict candidate gene(s) for targeted QTLs interval based on reference genome annotation information; (iv) develop KASP markers of the candidate gene(s) and verified by PG-RIL and a natural population consisted of 141 cultivar/lines for markerassisted selection in high-TKW wheat breeding.

\section{Results \\ Phenotypic performance and correlation analysis}

The 176 RIL population and their two parents P3228, G8901 were planted in four environments to identify stable and major QTLs for kernel-related traits. The means and ranges of four kernel-related traits (TKW, KL, $\mathrm{KW}$ and $\mathrm{KL} / \mathrm{W})$ are listed in Table 1. Compared with P3228, G8901 had wider KW, but shorter KL (Fig. 1 and Table 1). For the RIL population, the frequency of kernel traits in all environments and best linear unbiased predictors (BLUP) showed a continuous distribution with ranges from 27.33 to $44.97 \mathrm{~g}$ in TKW, 5.64 to $7.09 \mathrm{~mm}$ in $\mathrm{KL}$, 2.84 to $3.39 \mathrm{~mm}$ in KW and 1.78 to 2.43 in KL/W (Table 1 and Fig. 2). The Shapiro-Wilk test and Pearson's correlation coefficients of the four traits were calculated based on the BLUP data of four individual environments, indicating that TKW, KL, KW and KL/W showed normal distributions in multiple environments (Fig. 2 and Table 2). Moreover, TKW was positively correlated with KL and KW, and negatively correlated with KL/W (Table 2). The variance for genotype, environment and genotype $\times$ environment (GE) interaction effects were highly significant in TKW, KL, KW and KL/W (Additional file 1: Table S1). All the broad-sense heritability $(H)$ of four traits were higher than 0.60 (Table 2), indicating that these traits were mainly determined by genetic factors.

\section{QTL mapping}

A total of 47 putative QTLs were detected for TKW, KL, KW and KW/L (Figs. 3a-3d and Additional file 1: Table S2). Among them, 25, eight and 13 QTLs were located on the A, B and D genome, respectively. The single QTL explained $1.79-22.41 \%$ of the phenotypic variance with threshold log-of-odds (LOD) value ranging from 2.54 to 
Table 1 Phenotypes of the parents and PG-RIL population in this study

\begin{tabular}{|c|c|c|c|c|c|c|c|c|c|}
\hline \multirow[b]{2}{*}{ Trait } & \multirow[b]{2}{*}{ Env } & \multicolumn{2}{|c|}{ Parents } & \multicolumn{6}{|c|}{ PG-RILs } \\
\hline & & P3228 & G8901 & Min & Max & Mean & SD & CV(\%) & $H$ \\
\hline \multirow[t]{5}{*}{ TKW (g) } & E1 & 31.68 & 36.93 & 20.79 & 46.77 & 32.54 & 4.37 & 13.42 & 0.668 \\
\hline & E2 & 38.23 & 44.22 & 24.18 & 45.86 & 35.05 & 3.63 & 10.35 & \\
\hline & E3 & 41.13 & 46.81 & 28.22 & 49.87 & 37.72 & 3.79 & 10.05 & \\
\hline & E4 & 33.64 & 40.95 & 23.43 & 46.32 & 33.82 & 4.12 & 12.18 & \\
\hline & BLUP & 36.17 & 42.23 & 27.34 & 44.97 & 34.79 & 3.07 & 8.82 & \\
\hline \multirow[t]{5}{*}{$\mathrm{KL}(\mathrm{mm})$} & E1 & 6.70 & 6.36 & 5.53 & 7.22 & 6.33 & 0.31 & 4.85 & 0.859 \\
\hline & E2 & 6.67 & 6.29 & 5.50 & 7.04 & 6.25 & 0.29 & 4.61 & \\
\hline & E3 & 6.86 & 6.42 & 5.67 & 7.18 & 6.45 & 0.28 & 4.36 & \\
\hline & E4 & 6.78 & 6.46 & 5.62 & 7.21 & 6.45 & 0.29 & 4.45 & \\
\hline & BLUP & 6.75 & 6.38 & 5.64 & 7.09 & 6.37 & 0.26 & 4.15 & \\
\hline \multirow[t]{5}{*}{$\mathrm{KW}(\mathrm{mm})$} & E1 & 2.91 & 3.23 & 2.52 & 3.32 & 2.95 & 0.16 & 5.32 & 0.615 \\
\hline & E2 & 3.02 & 3.29 & 2.64 & 3.34 & 3.03 & 0.13 & 4.32 & \\
\hline & E3 & 3.33 & 3.59 & 2.92 & 3.77 & 3.30 & 0.14 & 4.21 & \\
\hline & E4 & 3.08 & 3.42 & 2.73 & 3.52 & 3.10 & 0.15 & 4.77 & \\
\hline & BLUP & 3.09 & 3.38 & 2.84 & 3.39 & 3.10 & 0.11 & 3.39 & \\
\hline \multirow[t]{5}{*}{$\mathrm{KL} / \mathrm{W}$} & E1 & 2.30 & 1.97 & 1.83 & 2.61 & 2.16 & 0.14 & 6.48 & 0.796 \\
\hline & E2 & 2.21 & 1.91 & 1.76 & 2.46 & 2.07 & 0.12 & 5.80 & \\
\hline & E3 & 2.06 & 1.79 & 1.68 & 2.27 & 1.97 & 0.12 & 6.09 & \\
\hline & E4 & 2.20 & 1.89 & 1.80 & 2.52 & 2.10 & 0.12 & 5.71 & \\
\hline & BLUP & 2.19 & 1.89 & 1.78 & 2.43 & 2.08 & 0.11 & 5.25 & \\
\hline
\end{tabular}

Notes: TKW, thousand kernel weight; KL, kernel length; KW, kernel width; KL/W, kernel length/kernel width ratio; Env, environment; Min, minimum; Max, Maximum; BLUP, best linear unbiased predictors mean

11 (Additional file 1: Table S2). Seventeen stable QTLs could be detected in more than two individual environments (Fig. 3a-e and Table 3).

A total of 19 QTLs for TKW were identified, of which 13 carried the favorable alleles from G8901 can increase the TKW, while the remaining six were from P3228 (Fig. 3a-d and Additional file 1: Table S2). In addition, five stable QTLs can be detected in at least two environments, including QTkw.cas-1A.2, QTkw.cas-4A, QTkw.cas-5D, QTkw.cs6A.1 and QTkw.cas-7D.2 (Table 3). Remarkably, the major stable QTL QTkw.cas-4A, located on chromosome arm $4 \mathrm{AL}$, can be repeatedly detected in all the environments and BLUP data, and phenotypic variance explained (PVE) ranged from 8.31 to $11.84 \%$ (Fig. $3 \mathrm{~b}-\mathrm{c}$ and Table 3 ). QTkw.cas-6A.1 can be detected in the three environments as well as BLUP data, and the PVE ranged from 6.52 to $12.73 \%$ (Fig. 3c and Table 3). The favorable allele of QTkw.cas-4A was derived from the parent G8901, while QTkw.cas-6A.1 was derived from the parent P3228. QTkw.cas-1A.2, QTkw.cas-5D and QTkw.cas-7D.2 were three stable QTLs, with PVE at 4.68-5.93\%, 3.28-4.28\% and $5.50-6.52 \%$, respectively (Table 3 ).

Ten QTLs for KL were detected, of which five QTLs (QKl.cas-1A.2, QKl.cas-1B, QKl.cas-2A, QKl.cas-4A and QKl.cas-7A.1) were significant in at least two environments
(Figs. 3a-d, Table 3 and Additional file 1: Table S2). The major QTL QKl.cas-2A was significant in two environments, explaining $8.40-10.28 \%$ of the phenotypic variance (Fig. $3 \mathrm{~b}$ and Table 3). Notably, the most stable QTL QKl.cas-4A was co-located with QTL QTkw.cas-4A for TKW (Fig. 3b and Table 3). Among the 10 QTLs for KL, six had additive effects from P3228 (Additional file 1: Table S2).

Eight QTLs for KW were identified on chromosomes 1A (two), 1B, 4B, 6A, 7A (two) and 7D, respectively (Figs. 3a-e, Table 3 and Additional file 1: Table S2). Among the three environments, the most stable QTL QKw.cas-6A in three environments was located on chromosome arm 6AS with PVE ranging from 5.43 to $9.85 \%$ (Fig. 3c and Table 3). This locus was co-located with the major QTL for TKW on 6AS (QTkw.cas-6A.1). The favorable alleles of the five QTLs (QKw.cas-1A.2, QKw.cas-1B, QKw.cas-7A, QKw.cas-7D.1 and $Q K$ w.cas-7D.2) were derived from the parent G8901 (Figs. 3a-e, Table 3 and Additional file 1: Table S2).

A total of 10 QTLs for KL/W were identified on chromosomes 1A, 1B, 2A, 5A (two), 5D, 7A (two) and 7D (two), with PVE of individual QTL ranging from 1.79 to $22.41 \%$ (Figs. 3a-d, Table 3 and Additional file 1: Table S2). Five QTLs (QKl/w.cas-1A, QKl/w.cas-2A, QKl/w.cas-5A.2, QKl/ w.cas-7A.1 and $Q K l / w . c a s-7 A .2)$ were found in at least two environments (Table 3). Among them, the major stable 


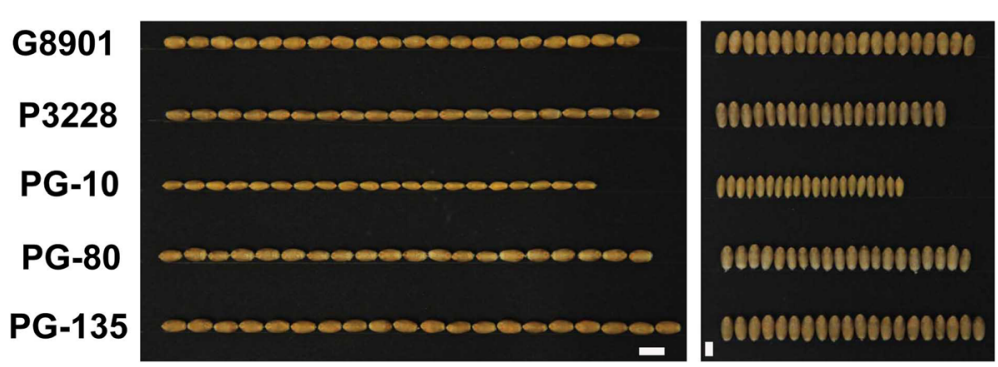

Fig. 1 Phenotypic characterization of two parents and some representative RIL

QTL QKl/w.cas-7A.1 can be detected in all the environments and BLUP data, explaining $3.85-13.84 \%$ of the phenotypic variance (Fig. 3d and Table 3). This QTL was co-located with QTLs for KW on chromosome 7A (QKw.cas-7A).

\section{Epistasis and QTL $\times$ environment interaction}

A total of 15 pairs of epistasis QTLs for TKW, KL, KW and $\mathrm{KW} / \mathrm{L}$ were detected, involving 30 QTLs on 15 chromosomes (Additional file 1: Table S3). Three pairs of epistasis interaction QTLs for TKW with PVE of 11.20, 7.10, and $8.93 \%$ were detected on chromosomes $1 \mathrm{~B} / 2 \mathrm{D}, 4 \mathrm{D} / 6 \mathrm{D}$, and $5 \mathrm{~A} / 6 \mathrm{D}$, respectively, indicating that the interactions between those QTLs had no significant main effect on TKW (Additional file 1: Table S3). Three pairs of epistasis interaction sites of $\mathrm{KL}$ were detected, among which the interactions on chromosomes $4 \mathrm{~A} / 3 \mathrm{~B}$ was between the major and non-major QTLs, while the interactions on $2 \mathrm{D} / 3 \mathrm{~A}$ and $6 \mathrm{~B} /$ 6D were between non-majors, and all of the three QTLs could increase KL (Additional file 1: Table S3). Four pairs of epistasis interactional QTLs for KW were detected, and they were all interactional between non-major QTLs. The two combinations of $3 \mathrm{~B} / 6 \mathrm{~A}$ and $5 \mathrm{~B} / 6 \mathrm{D}$ could increase the $\mathrm{KW}$, while the two combinations of $4 \mathrm{~B} / 6 \mathrm{~B}$ and $5 \mathrm{D} / 6 \mathrm{~B}$ could decrease the KW. Five pairs of epistasis interactional QTLs for KL/W were detected, all of which were interactional between non-major QTLs. The two combinations of $6 \mathrm{D} / 6 \mathrm{D}$ and $1 \mathrm{~B} / 6 \mathrm{D}$ could reduce $\mathrm{KL} / \mathrm{W}$, while the other three combinations could increase KL/W.

QTL $\times$ environment $(\mathrm{QE})$ interactions were detected at 43 loci for TKW, KL, KW and KW/L (Additional file 1: Table S4). They overlapped with 47 putative QTLs of four traits, indicating that the TKW, KL, KW and KL/W were affected by environment. Among them, the largest environmental effect was detected in the interval $A X-109416575-A X$ 108738265 (PVE $(\mathrm{AbyE})=21.93 \%$ ), indicating that the major QTLs QTkw.cas-4A and QKl.cas-4A for TKW and $K L$, respectively, were significantly affected by the environment (Additional file 1: Table S4). Ten pairs of epistasis interactions were detected for additive-additive-environment (AAE), including three, one, three and three pairs of epistasis QTLs for TKW, KL, KW and KL/W, respectively (Additional file 1: Table S3).
QTL analysis for TKW conditioned on kernel-related traits To dissect genetic effects of the KL, KW and KL/W on the expression of QTLs for TKW, conditional QTL analysis were conducted. After conditioned on KL, KW or KL/W, a total of 23 conditional QTLs comprising 47 QTL $\times$ environments were detected for TKW (Additional file 1: Table S5). Among them, 19 QTLs were identified as unconditional analysis, while the other 10 QTLs were newly detected, with four QTLs identified in at least two environments (Additional file 1: Table S5).

The QTLs QTkw.cas-2A.1, QTkw.cas-4A and QTkw.cas$4 D$ were detected when TKW was conditioned on KW and KL/W instead of KL (Table 4 and Additional file 1: Table S5). This result indicated that these QTLs may be associated with $\mathrm{KL}$, but independent of KW and KL/W. Four QTLs (QTkw.cas-5A, QTkw.cas-6A.1, QTkw.cas-7A and QTkw.cas-7D.2) were identified to be associated with $\mathrm{KW}$, but independent of KL and KL/W (Table 4 and Additional file 1: Table S5). The QTL QTkw.cas-1A.2, was detected when TKW was conditioned on $\mathrm{KL}$, but absent when conditioned on KW or KL/W (Table 4), suggesting that it may be independent of KL, but was associated with either one or both of KW and KL/W. The stable QTL QTkw.cas-5D was not detected when TKW was conditioned on KL, KW or KL/W (Table 4).

\section{Important QTL clusters}

A total of seven QTL clusters were identified, all of them were related to more than one trait (Fig. 3a-d and Table 5). Three intervals harboring various QTLs can be identified in at least three environments (Fig. 3a-d, Tables 3 and 5). The interval $A X-110540586-A X-108840708$ on chromosome 4A affected TKW and KL across all the four environments and BLUP data, and the additional effects were derived from G8901 (Fig. 3a-d, Tables 3 and 5). The interval $A X$ $109892808-A X-110438513$ on chromosome 6A affected TKW and KW across the three environments and BLUP data, with P3228 conferring the favorite allele (Fig. 3c and Table 5). The interval $A X-111061288-A X-111184541$ on chromosome 7D showed significant effects on TKW and KW across three environments and BLUP data and on KL/ $\mathrm{W}$ in one environment and BLUP data (Table 5 and Fig. 

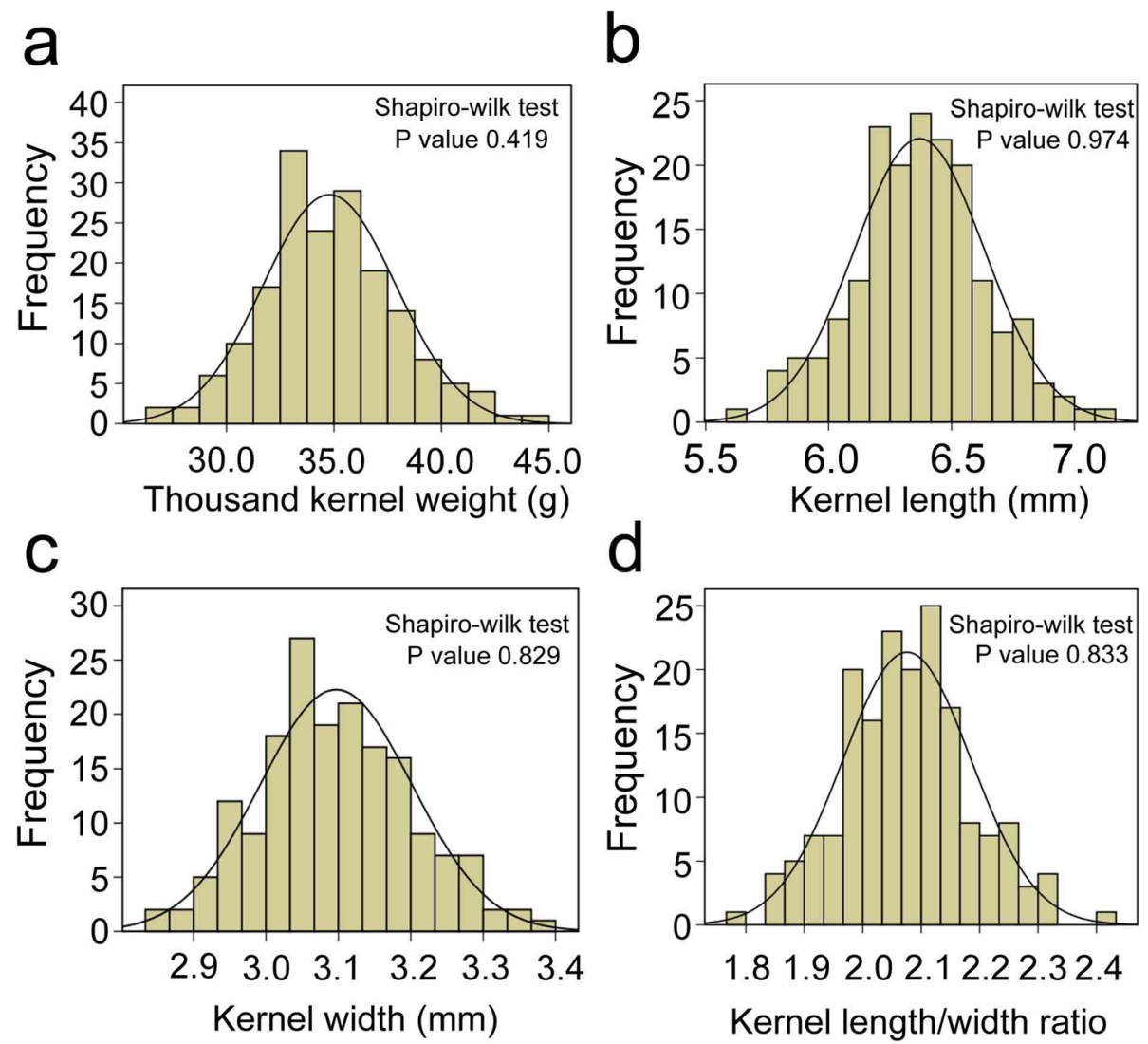

Fig. 2 Frequency distribution of four kernel traits in RIL population in BLUP data. a Thousand kernel weight. b Kernel length. c Kernel width. $\mathbf{d}$ Kernel length/width

3d). In this interval, the G8901-derived allele increased TKW and KW and decreased KL/W (Table 3).

Predicting of candidate gene TaFT-D1 for QTLs QTkw.cas7D.2 and QKw.cas -7D.1

The two stable QTLs, QTkw.cas-7D.2 and QKw.cas7D.1, was delimited by the markers $A X-110826147$ and $A X-111359934$ (Fig. 3d), and the peak interval were colocated between the markers $A X-111061288$ and $A X$ 111184541 (Table 3 and Fig. 3d-e). Collinearity analysis indicated that the genetic map of PG-RIL and the physical map of Chinese Spring reference genome V1.0 show perfect collinearity in the chromosomes 7DS region (Additional file 2: Fig. S1). To investigate the physical intervals of QTLs QTkw.cas-7D.2 and QKw.cas-7D.1, we aligned the markers $A X-110826147$ and $A X-111359934$ to Chinese Spring reference genome V1.0 [49]. The results showed that the physical interval of QTLs QTkw.cas-7D.2 and QKw.cas-7D.1 is mapped to the 65.50-69.32 Mb position on chromosome arm 7DS which contained 47 high confidence genes (Table 3 and Additional file 2: Table. S2).

Subsequently, we annotated 47 genes in the $3.82 \mathrm{Mb}$ region (Additional file 2: Table. S3). Among them, TaFT-D1 (TraesCS7D02G111600), a homolog of Arabidopsis FLOWERING LOCUS T, was considered as the candidate gene for QTkw.cas-7D.2 and QKw.cas-7D.1 (Additional file 1: Tables S6). Then, we designed genome-specific

Table 2 Correlation coefficients among the kernel traits of PG-RIL population in four environments

\begin{tabular}{|c|c|c|c|c|c|c|c|c|c|c|c|c|c|c|c|}
\hline \multirow[t]{2}{*}{ Trait } & \multicolumn{3}{|l|}{ BLUP } & \multicolumn{3}{|l|}{ E1 } & \multicolumn{3}{|l|}{ E2 } & \multicolumn{3}{|l|}{ E3 } & \multicolumn{3}{|l|}{ E4 } \\
\hline & TKW & $\mathrm{KL}$ & KW & TKW & $\mathrm{KL}$ & KW & TKW & $\mathrm{KL}$ & KW & TKW & $\mathrm{KL}$ & KW & TKW & $\mathrm{KL}$ & KW \\
\hline$\overline{\mathrm{KL}}$ & $0.458^{* *}$ & & & $0.387^{* *}$ & & & $0.476^{* *}$ & & & $0.432^{* *}$ & & & $0.469^{* *}$ & & \\
\hline $\mathrm{KW}$ & $0.823^{* *}$ & 0.085 & & $0.901^{* *}$ & 0.133 & & $0.792^{* *}$ & 0.070 & & $0.788^{* *}$ & 0.0288 & & $0.874^{* *}$ & $0.194^{* *}$ & \\
\hline $\begin{array}{l}\mathrm{KL} / \\
\mathrm{W}\end{array}$ & $-0.235^{* *}$ & $0.708^{* *}$ & $-0.641^{* *}$ & $\overline{-}^{0.515^{* *}}$ & $0.536^{* *}$ & $-0.763^{* *}$ & $0.204^{* *}$ & $0.781^{* *}$ & $-0.566^{* *}$ & $\overline{-}^{-} 243^{* *}$ & $0.707^{* *}$ & $-0.684^{* *}$ & $-\overline{-}^{* 356^{* *}}$ & $0.600^{* *}$ & $-0.665^{* *}$ \\
\hline
\end{tabular}

Note: ${ }^{*}$ significant at $P<0.05$ level; ${ }^{* *}$ significant at $P<0.01$ level 


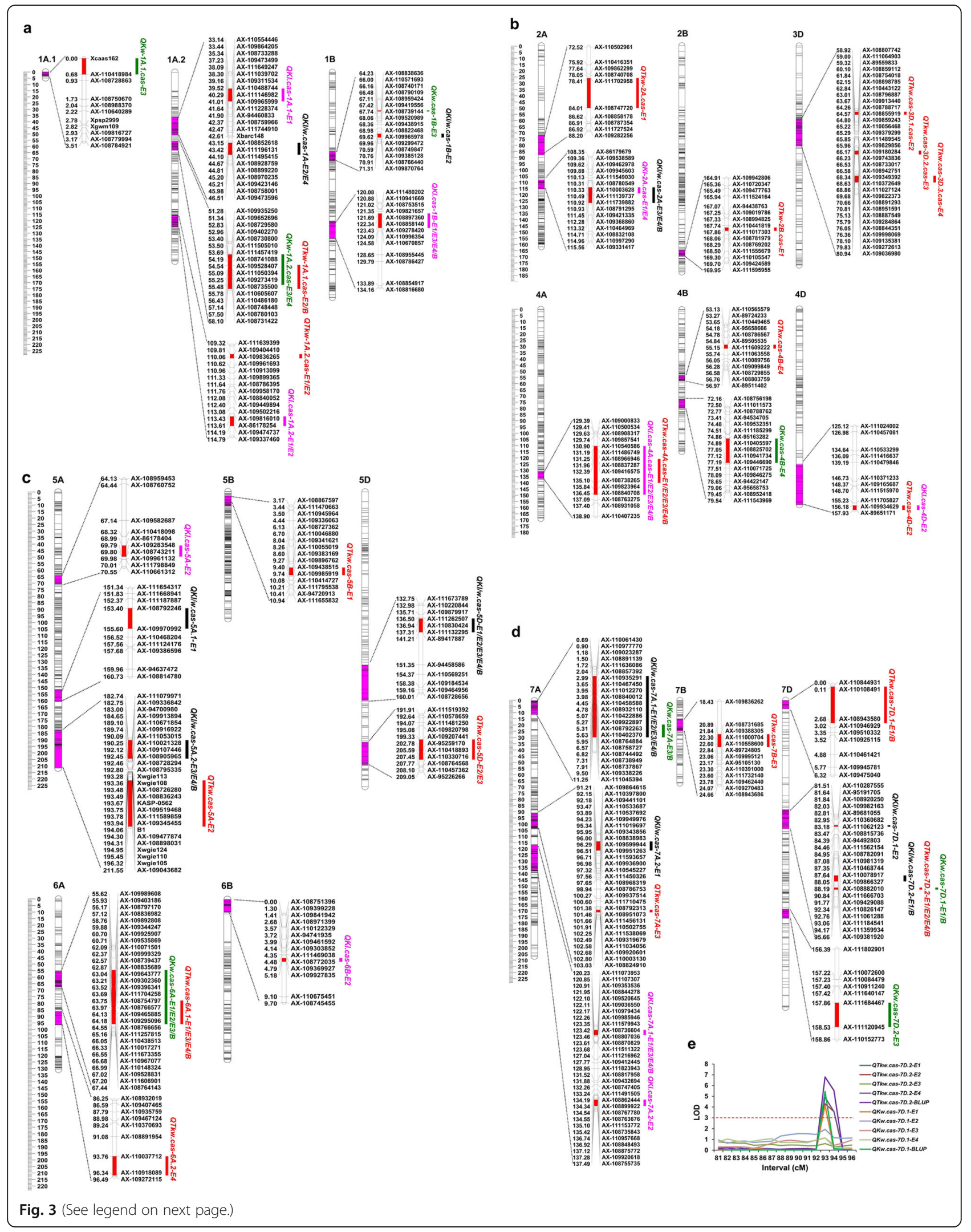


(See figure on previous page.)

Fig. 3 Genetic and physical locations of QTL regions associated with TKW, KL, KW and KL/W. a QTLs located on the chromosome 1A and 1B. b QTLS located on the chromosome 2A, 2B, 3D, 4A, 4B and 4D. c QTLs located on the chromosome 5A, 5B, 5D, 6A and 6B. (d) QTLs located on the chromosome 7A, 7B and 7D. (e) LOD curves for the QTLs QTkw.cas-7D.2 and QKw.cas-7D.1 on chromosome 7D. Uniform centimorgan (cM) scales are shown on the left. Physical maps are shown on the right of each genetic map. QTLs are indicated on the right side of each chromosome. For QTLs detected in different environments, a slash is inserted to distinguish the environments. The codes E1, E2, E3, E4 and B represent QTLs detected in 2013LC, 2014LC, 2015LC, 2016LC environments and BLUP data, respectively. Red, pink, green, black colors represent QTLs conferring TKW, KL, KW and KLW, respectively

primers for sequencing to analyse the genome sequence of TaFT-D1 from G8901 and P3228 (Additional file 1: Tables S9), and found that there was a 1 bp deletion at position +840 in the third exon of TaFT-D1 in P3228. Protein sequence alignment revealed that this deletion caused frameshift mutation with loss function of the TaFT-D1 protein in P3228 (Additional file 2: Fig. S2). We further analyzed the expression profiles of 47 candidate genes in different tissues using the Chinese Spring cv-1 development (pair) database [50]. As shown in Additional file 2: Fig. S3, the expression of TaFT-D1 was highest in leaves and young spikes, slightly lower in stems and substantially lower in root and developing grain.

\section{Development of KASP markers and analysis for alleles of TaFT-D1}

Two SNPs markers $(A X-111061288$ and $A X-111184541)$ closely linked to the two stable QTLs (QTkw.cas-7D.2 and QKw.cas-7D.1) and $1 \mathrm{bp}$ InDel of TaFT-D1 were further converted to KASP markers (Fig. 4a, Additional file 2: Fig. S4 and Additional file 1: Tables S9). After screening PG-RIL and a natural population consisted of 141 cultivar/ lines using these KASP markers, we found that the KASP marker of TaFT-D1 was co-segregated with SNPs marker $A X-111184541$. This result further proved that TaFT-D1 was an important candidate gene for the QTkw.cas-7D.2 and QKw.cas-7D.1. Furthermore, two-tailed t test was performed between the InDel of TaFT-D1 and four kernelrelated traits collected from multiple environments. The results showed that the InDel of TaFT-D1 was significantly correlated with TKW, KW and KL/W but not with KL for PG-RIL (Fig. 4b-e). For the natural population consisted of 141 cultivar/lines, the InDel of TaFT-D1 was associated with TKW and KW in the three environments, except that no significant differences were observed in the KL and KL/ W of G8901-allele (TaFT-D1(G)-allele) and P3228-allele (TaFT-D1(-)-allele) plants (Figs. 4f-i). The mean TKW of TaFT-D1(G)-allele was significantly higher than those of the TaFT-D1(-)-allele (mean $4.91 \mathrm{~g}$ higher in 2013-2014, 5.21 g higher in 2014-2015, $2.87 \mathrm{~g}$ higher in 2015-2016 and $1.58 \mathrm{~g}$ higher in 2016-2017).

\section{TaFT-D1(G)-allele underwent positive selection during Chinese wheat breeding}

To determine whether the two TaFT-D1 alleles were subjected to selecting, we investigated the geographic distribution of the TaFT-D1 alleles in 150 Chinese wheat landraces and 172 modern cultivars. The Chinese wheat production area is divided into 10 agro-ecological wheat production regions according to environment, type of cultivars and growing season $[51,52]$. Compared with landraces, the proportion of TaFT-D1(G)-allele in modern cultivars was higher in the seven agro-ecological wheat production regions (except for regions IV, VIII and IX), suggesting that TaFT-D1(G)-allele have undergone positive selection during wheat breeding process (Fig. 5a and b). This confirmed that the favorable TaFT-D1(G)-allele can be used in different wheat production regions.

\section{Discussion}

Unconditional QTLs and conditional QTLs effects

Previous researches have shown that the combination of QTL mapping and conditional genetic analysis enable the identification of the influence of one trait on another [22, 28]. In the current study, we dissected QTLs based on TKW values conditioned on KL, KW and KL/W to study the genetic basis of TKW on QTL expression. When conditioned on KW, four conditional stable QTLs (QTkw.cas1A.2, QTkw.cas-5D, QTkw.cas-6A.1, QTkw.cas-7D.2) account for TKW, while two (QTkw.cas-4A and QTkw.cas$5 D$ ) on KL (Table 4). Notably, QTkw.cas-5D was not detected when TKW was conditioned on KL or KW (Table 4). The total PVE of the four QTLs conditioned on KW was significantly higher than the two on KL, indicating that KW contributes more than KL to TKW in the PGRIL population (Table 4). The unconditional QTL analysis showed that the major QTL QTkw.cas-4A on chromosome 4A was co-located with QTL QKl.cas-4A for KL, with G8901-derived allele increasing both TKW and KL (Table 3 and Fig. 3b). Using conditional QTL analysis, we found that the QTkw.cas-4A was entirely contributed by $\mathrm{KL}$, partially by KW and entirely independent by KL/W (Table 4). Combining unconditional QTL with conditional QTLs analysis, the effect of increasing TKW of QTkw.cas$4 A$ was identified to be mainly caused by the KL. Using the same analysis methods, we concluded that the effects of increasing TKW of QTkw.cas-6A and QTkw.cas-7D were mainly contributed by the KW. The results should be valuable for dissecting the genetic basis of TKW and the genetic contribution of kernel related traits to TKW at individual QTL level in wheat. 
Table 3 Stable QTLs for thousand kernel weight, Kernel length, Kernel width, Kernel length/width traits in the PG-RIL population

\begin{tabular}{|c|c|c|c|c|c|c|c|}
\hline Trait & QTL & Left Markers & Interval (cM) & $E$ & PVE $\%$ & Add & Reference \\
\hline \multirow[t]{19}{*}{ TKW } & \multirow[t]{4}{*}{ QTkw.cas-1A.2 } & \multirow{4}{*}{$\begin{array}{l}A X-109528407 \\
-A X-108731422\end{array}$} & \multirow[t]{4}{*}{$54.55-58.10$} & E2 & 5.93 & -0.92 & \multirow[t]{4}{*}[8,36,43]{} \\
\hline & & & & E3 & 4.68 & -0.84 & \\
\hline & & & & E4 & 6.92 & -1.13 & \\
\hline & & & & BLUP & 5.35 & -0.74 & \\
\hline & \multirow[t]{5}{*}{ QTkw.cas-4A } & \multirow{5}{*}{$\begin{array}{l}A X-109416575 \\
-A X-108738265\end{array}$} & \multirow[t]{5}{*}{ 132.39-135.10 } & E1 & 10.59 & -1.428 & \multirow[t]{7}{*}[40,44]{} \\
\hline & & & & E2 & 8.31 & -1.051 & \\
\hline & & & & E3 & 11.84 & -1.311 & \\
\hline & & & & E4 & 10.69 & -1.354 & \\
\hline & & & & BLUP & 13.17 & -1.162 & \\
\hline & \multirow[t]{2}{*}{ QTkw.cas-5D } & \multirow{2}{*}{$\begin{array}{l}A X-109207441 \\
-A X-110418893\end{array}$} & \multirow[t]{2}{*}{ 199.33-205.59 } & E2 & 4.28 & 0.777 & \\
\hline & & & & E3 & 3.28 & 0.699 & \\
\hline & \multirow[t]{4}{*}{ QTkw.cas-6A.1 } & \multirow{4}{*}{$\begin{array}{l}A X-108835689 \\
-A X-110438513\end{array}$} & \multirow[t]{4}{*}{$62.87-66.05$} & E1 & 6.52 & 1.137 & \multirow[t]{4}{*}[45,46]{} \\
\hline & & & & E3 & 11.38 & 1.308 & \\
\hline & & & & E4 & 12.73 & 1.526 & \\
\hline & & & & BLUP & 11.77 & 1.102 & \\
\hline & \multirow[t]{4}{*}{ QTkw.cas-7D.2 } & \multirow{4}{*}{$\begin{array}{l}A X-111061288 \\
-A X-111184541\end{array}$} & \multirow[t]{4}{*}{$92.76-93.06$} & E1 & 6.52 & -1.150 & \multirow[t]{4}{*}[39,43,47]{} \\
\hline & & & & E2 & 5.50 & -0.895 & \\
\hline & & & & E4 & 6.09 & -1.069 & \\
\hline & & & & BLUP & 6.22 & -0.811 & \\
\hline \multirow[t]{17}{*}{$\mathrm{KL}$} & \multirow[t]{2}{*}{ QKI.cas-1A.2 } & \multirow{2}{*}{$\begin{array}{l}A X-86178254 \\
-A X-109474737\end{array}$} & \multirow[t]{2}{*}{$113.61-114.19$} & E1 & 4.29 & 0.061 & \\
\hline & & & & E2 & 5.63 & 0.066 & \\
\hline & QKI.cas-1B & $A X-108897360$ & $66.00-66.16$ & E1 & 6.63 & 0.075 & {$[36]$} \\
\hline & & $-A X-110996354$ & & E3 & 8.16 & 0.073 & \\
\hline & & & & E4 & 3.74 & 0.053 & \\
\hline & & & & BLUP & 7.38 & 0.071 & \\
\hline & QKI.cas-2A & AX-108791295 & 110.93-111.45 & E1 & 14.35 & 0.110 & {$[37]$} \\
\hline & & $-A X-109421333$ & & E4 & 16.65 & 0.111 & \\
\hline & QKI.cas-4A & AX-110540586 & 130.90-136.45 & E1 & 7.48 & -0.080 & \\
\hline & & $-A X-108840 / 08$ & & E2 & 8.46 & -0.081 & \\
\hline & & & & E3 & 8.09 & -0.073 & \\
\hline & & & & E4 & 9.66 & -0.085 & \\
\hline & & & & BLUP & 9.00 & -0.078 & \\
\hline & QKI.cas-7A.1 & AX-109353536 & $120.91-122.10$ & E1 & 5.78 & -0.070 & \\
\hline & & $-A$ & & E3 & 7.24 & -0.069 & \\
\hline & & & & E4 & 5.70 & -0.065 & \\
\hline & & & & BLUP & 7.24 & -0.070 & \\
\hline $\mathrm{KW}$ & QKw.cas-1A.2 & $A X-109402270$ & $52.96-57.14$ & E3 & 5.55 & -0.031 & \\
\hline & & $-A X-108748448$ & & E4 & 10.14 & -0.047 & \\
\hline & QKw.cas-6A & AX-109892808 & $58.76-66.05$ & E1 & 5.43 & 0.037 & {$[45,48]$} \\
\hline & & $-A X-110438513$ & & E2 & 9.85 & 0.040 & \\
\hline & & & & E3 & 7.31 & 0.036 & \\
\hline & & & & BLUP & 7.98 & 0.030 & \\
\hline
\end{tabular}


Table 3 Stable QTLs for thousand kernel weight, Kernel length, Kernel width, Kernel length/width traits in the PG-RIL population (Continued)

\begin{tabular}{|c|c|c|c|c|c|c|c|}
\hline Trait & QTL & Left Markers & Interval (cM) & $E$ & PVE\% & Add & Reference \\
\hline \multirow[t]{18}{*}{$\mathrm{KL} / \mathrm{W}$} & \multirow[t]{2}{*}{ QKI/W.cas-1A } & \multirow{2}{*}{$\begin{array}{l}A X-111196131 \\
-A X-108970235\end{array}$} & \multirow[t]{2}{*}{$43.42-45.20$} & E2 & 22.41 & 0.093 & \\
\hline & & & & E4 & 5.22 & 0.027 & \\
\hline & \multirow[t]{3}{*}{ QKI/w.cas-2A } & \multirow{3}{*}{$\begin{array}{l}A X-108791295 \\
-A X-109368860\end{array}$} & \multirow[t]{3}{*}{$110.93-112.28$} & E3 & 11.60 & 0.040 & \\
\hline & & & & E4 & 11.03 & 0.039 & \\
\hline & & & & BLUP & 10.90 & 0.037 & \\
\hline & \multirow[t]{3}{*}{ QKI/w.cas-5A.2 } & \multirow{3}{*}{$\begin{array}{l}A X-94700980 \\
-A X-110671854\end{array}$} & \multirow[t]{3}{*}{ 183.00-189.10 } & E3 & 8.32 & -0.034 & \\
\hline & & & & E4 & 7.49 & -0.032 & \\
\hline & & & & BLUP & 6.27 & -0.028 & \\
\hline & \multirow[t]{5}{*}{ QKI/w.cas-5D } & \multirow{5}{*}{$\begin{array}{l}A X-110830424 \\
-A X-89417887\end{array}$} & \multirow[t]{5}{*}{$136.94-141.21$} & E1 & 6.70 & 0.039 & \\
\hline & & & & E2 & 3.77 & 0.038 & \\
\hline & & & & E3 & 5.60 & 0.028 & \\
\hline & & & & E4 & 5.58 & 0.028 & \\
\hline & & & & BLUP & 8.26 & 0.032 & \\
\hline & \multirow[t]{5}{*}{ QKI/w.cas-7A.1 } & \multirow{5}{*}{$\begin{array}{l}A X-111636086 \\
-A X-109338226\end{array}$} & \multirow[t]{5}{*}{$1.720-9.50$} & E1 & 13.84 & 0.055 & \\
\hline & & & & E2 & 3.85 & 0.038 & \\
\hline & & & & E3 & 12.25 & 0.041 & \\
\hline & & & & E4 & 8.18 & 0.034 & \\
\hline & & & & BLUP & 13.23 & 0.041 & \\
\hline
\end{tabular}

Notes: E: environments, BLUP: best linear unbiased predictors, PVE: phenotypic variance explained, Add: additive effect

\section{QTL comparison}

To date, a large number of QTLs for TKW and kernel morphological traits have been mapped in common wheat $[45,48]$. To investigate whether there were overlapping QTLs in different genetic backgrounds, we compared the QTLs interval in this study with those in the previous studies. Some stable QTLs have been reported in the previous studies. For example, the interval $A X-108835689$ $A X-110438513$ on chromosome 6A contained QTkw.cas$6 A .1$ and $Q K w$.cas-6A, corresponding to the reported QTLs for kernel weight in different RIL population [4446]. The gene $T a G W 2-A 1$ was also located in this interval, and it affects TKW by regulating the KW of bread wheat $[13,52]$. It was also reported that the major stable QTLs QTkw.cas-4A and QKl.cas-4A were in the interval $A X$ 108738265-AX-109416575 (Table 5), overlapping with the locus for TKW in the previous study [40, 47]. The QTL QTkw.cas-7D in the interval $A X-111061288-A X-$ 111184541 on chromosome 7D has also reported previously [39, 43, 53, 54]. Therefore, these important QTLs that were not affected by genetic background are important selection targets in wheat breeding.

\section{Advantages of high-density genetic maps}

Previous genetic maps were mainly constructed by gelbased markers. Moreover, the confidence intervals associated with detected QTLs were relatively large and the numbers of markers was limited, which restricted further fine mapping of QTLs and their applications in breeding [27, 38]. Compared with gel-based markers, high-density SNP arrays have the advantage of abundant markers and can further reduce the confidence interval for QTL localization. In this study, we used the wheat $660 \mathrm{~K}$ high-density SNP chips to screen the PG-RIL population, and found that the confidence interval for most QTLs was less than $3 \mathrm{cM}$ (Table 3 and Additional file 1: Table S2). Furthermore, the SNP markers in the confidence interval have clear base sequence and position information, which is effective for fine mapping using the reference genome [27]. For instance, the stable QTL QTkw.cas-7D.2 and QKw.cas-7D.1 were colocated in interval between $92.756-93.059 \mathrm{cM}$, and the physical interval of the Chinese Spring reference genome V1.0 is 65.50-69.32 Mb (Table 3 and Fig. 3).

\section{Functional prediction of candidate genes for QTkw.cas-7D.2 and QKw.cas-7D.1}

In crops, genes that regulated flowering have diverse functions, some affecting the yield-related traits [54]. Kernel weight can be manipulated by altering the duration of kernel filling, which is greatly influenced by flowering-related genes. For instance, overexpression of TaGW8, the positive regulator of cell proliferation and grain filling, results in early flowering and enhanced kernel width and yield in wheat $[55,56]$. Overexpression of TaZIM-A1 represses the expression of TaFT1, leading to a delay in heading date and decreased TKW in common wheat [57]. In the present 
Table 4 Unconditional and conditional stable QTLs for TKW in wheat

\begin{tabular}{|c|c|c|c|c|c|c|c|c|c|c|c|c|c|}
\hline \multirow[t]{3}{*}{ QTL } & \multirow[t]{3}{*}{ Intervals marker } & \multirow{2}{*}{\multicolumn{3}{|c|}{$\begin{array}{l}\text { Unconditional QTL } \\
\text { TKW }\end{array}$}} & \multicolumn{9}{|c|}{ Conditional QTL } \\
\hline & & & & & \multicolumn{3}{|c|}{ TKW|KL } & \multicolumn{3}{|c|}{ TKW|KW } & \multicolumn{3}{|c|}{$\mathrm{TKW} /(\mathrm{KL} / \mathrm{W})$} \\
\hline & & E & PVE\% & Add & E & PVE\% & Add & $E$ & PVE\% & Add & E & PVE\% & Add \\
\hline \multirow[t]{3}{*}{ QTkw.cas-1A.2 } & $A X-109528407-A X-108731422$ & E2 & 5.927 & -0.915 & & & & & & & & & \\
\hline & & E3 & 4.684 & -0.836 & & & & & & & & & \\
\hline & & E4 & 6.924 & -1.127 & E4 & 5.695 & $-0.893 b$ & & & & & & \\
\hline \multirow[t]{4}{*}{ QTkw.cas-4A } & $A X-109416575-A X-108738265$ & E1 & 10.586 & -1.428 & & & & E1 & 9.033 & $-0.654 \mathrm{~b}$ & E1 & 8.936 & $-1.372 \mathrm{a}$ \\
\hline & & E2 & 8.310 & -1.051 & & & & & & & E2 & 7.278 & -0.999 a \\
\hline & & E3 & 11.837 & -1.311 & & & & E3 & 6.509 & $-0.562 b$ & E3 & 11.238 & $-1.191 \mathrm{a}$ \\
\hline & & E4 & 10.687 & -1.354 & & & & E4 & 9.763 & $-0.687 \mathrm{~b}$ & E4 & 12.060 & $-1.301 \mathrm{a}$ \\
\hline \multirow[t]{2}{*}{ QTkw.cas-5D } & $A X-109207441-A X-110418893$ & E2 & 4.280 & 0.777 & & & & & & & & & \\
\hline & & E3 & 3.280 & 0.699 & & & & & & & & & \\
\hline \multirow[t]{3}{*}{ QTkw.cas-6A.1 } & $A X-108835689-A X-110438513$ & E1 & 6.515 & 1.137 & E1 & 5.812 & $0.989 b$ & & & & E2 & 6.208 & $0.832 \mathrm{~b}$ \\
\hline & & E3 & 11.380 & 1.308 & E3 & 11.609 & $1.257 \mathrm{a}$ & & & & & & \\
\hline & & E4 & 12.727 & 1.526 & E4 & 11.069 & $1.244 b$ & & & & E4 & 9.719 & $1.171 \mathrm{~b}$ \\
\hline \multirow[t]{4}{*}{ QTkw.cas-7D.2 } & $A X-111061288-A X-1111184541$ & E1 & 6.520 & -1.150 & E1 & 11.086 & $-1.381 c$ & & & & & & \\
\hline & & E2 & 5.502 & -0.895 & E2 & 7.700 & $-1.014 c$ & & & & & & \\
\hline & & & & & E3 & 4.806 & $-0.819 d$ & & & & & & \\
\hline & & E4 & 6.091 & -1.069 & E4 & 6.946 & $-0.997 a$ & & & & E4 & 4.649 & $-0.820 \mathrm{~b}$ \\
\hline
\end{tabular}

Note: adenotes the additive effect of a conditional QTL, in absolute values, that reduces or increase less than $10 \%$ compared to the corresponding unconditional QTL ${ }^{b}$ denotes the additive effect of a conditional QTL, in absolute values, that reduces more than $10 \%$ compared to the corresponding unconditional QTL 'denotes the additive effect of a conditional QTL, in absolute values, that increase more than $10 \%$ compared to the corresponding unconditional QTL. ${ }^{\mathrm{d}}$ denotes the QTL couldn't be detected in unconditional analysis, but can be detected in conditional analysis

$(+)$ indicates that the most favorable allele is derived from the parent P3228, (-) indicates that the most favorable allele is derived from the parent G8901. E and numerals in parentheses indicate the environment in which the QTL was detected and the percentage of phenotypic variance explained (PVE) by the additive effects of the mapped QTLs, respectively

study, the stable QTLs QTkw.cas-7D.2 and QKw.cas-7D.1 were delimited to the $3.82 \mathrm{Mb}$ physical interval with 47 high-confidence genes (Additional file 1: Table S6). Among them, compared with G8901, frameshift mutation of TaFTD1 in P3228 leads to loss of protein function (Additional file 2: Fig. S2). TaFT1, a homolog gene of Arabidopsis FLOWERING LOCUS $T$, is a major gene that regulates wheat flowering $[58,59]$. It has diverse functions on regulating different reproductive traits, such as flowering time, spike development and seed development [60, 61]. The loss function of TaFT-D1 in P3228-allele lines resulted in delayed flowering and decreased TKW, while the high expression of TaFT-D1 in the G8901-allele lines leads to accelerated flowering time and increased TKW.

Table 5 Characterization of QTL clusters for kernel traits in this study

\begin{tabular}{|c|c|c|c|c|c|c|}
\hline Clusters & Chromosomes & Intervals marker & Intervals (cM) & QTL included & $\begin{array}{l}\text { No of } \\
\text { QTLs }\end{array}$ & $\begin{array}{l}\text { Traits (additive effect, } \\
\text { number of environments) }^{\mathrm{a}}\end{array}$ \\
\hline$\overline{C 1}$ & $1 \mathrm{~A}$ & $A X-111196131-A X-108731422$ & $43.42-58.07$ & $\frac{Q T K w . c a s-1 A .2,}{Q \text { QKI/w.cas-1A }}, \underline{\text { QKw.cas-1A.2, }}$ & 3 & 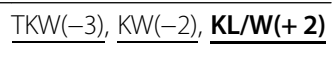 \\
\hline $\mathrm{C} 2$ & $2 \mathrm{~A}$ & $A X-108791295-A X-109368860$ & $110.93-112.28$ & QKI.cas-2A, QKI/w.cas- $2 A$ & 2 & $\underline{\mathrm{KL}(+2)}, \underline{\mathrm{KL} / \mathrm{W}(+2)}$ \\
\hline C3 & $4 \mathrm{~A}$ & $A X-110540586-A X-108840708$ & $130.91-136.45$ & QTkw.cas- $4 A$, QKl.cas- $4 A$ & 2 & $\underline{\operatorname{TKW}(-4)}, \underline{K L(-4)}$ \\
\hline C4 & $4 \mathrm{D}$ & $A X-109934629-A X-89651171$ & $156.18-157.93$ & QTkw.cas-4D, QKI.cas-4D & 2 & $\operatorname{TKW}(+1), \mathrm{KL}(+1)$ \\
\hline C5 & $6 \mathrm{~A}$ & $A X-109892808-A X-110438513$ & $58.76-66.05$ & QTkw.cas-6A.1, QKw.cas-6A & 2 & $\underline{\operatorname{TKW}(+3)}, \underline{K W(+3)}$ \\
\hline C6 & $7 \mathrm{~A}$ & $A X-111636086-A X-109338226$ & $1.72-9.50$ & QKw.cas-7A, QKI/w.cas-7A.1 & 2 & $\mathrm{KW}(-1), \underline{\mathrm{KL} / \mathrm{W}(+4)}$ \\
\hline $\mathrm{C7}$ & $7 D$ & $A X-111666703-A X-111184541$ & $90.84-93.06$ & $\frac{\text { QTKw.cas-7D.2, }}{\text { QKI/w.cas-7D.2 }}$ & 3 & $\underline{\operatorname{TKW}(-3)}, \operatorname{KW}(-1), \mathrm{KL} / \mathrm{W}(+1)$ \\
\hline
\end{tabular}

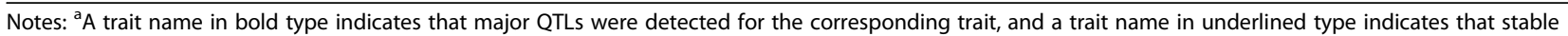
QTLs were detected for the corresponding traits. (+) indicates that the most favorable allele is derived from the parent P3228, $(-)$ indicates that the most favorable allele is derived from the parent G8901 
a

Allelic Discrimination

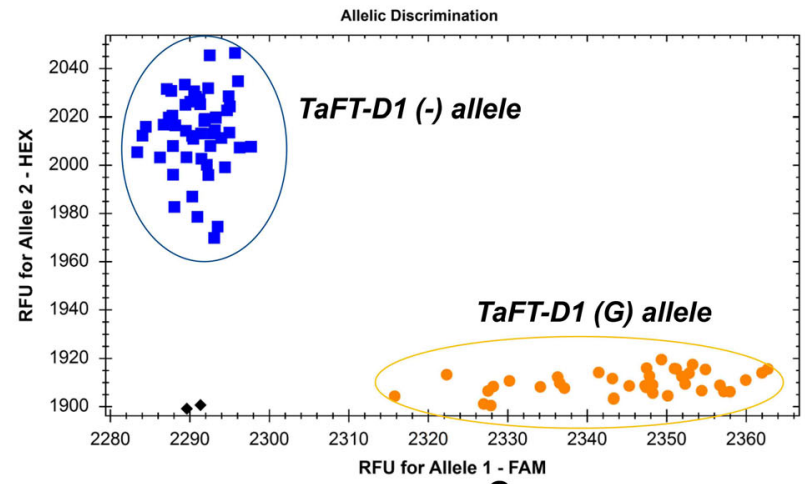

b

口TaFT-D1 (-) allele

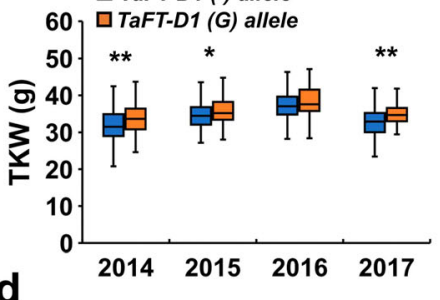

C $^{1-F A M}$

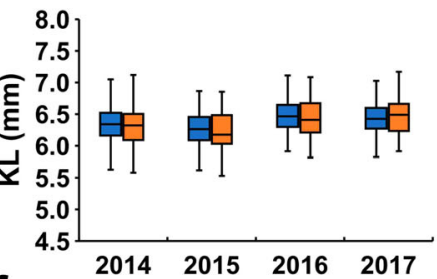

f

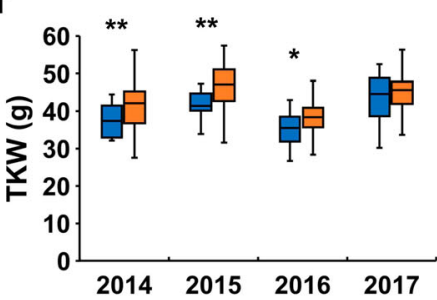

h

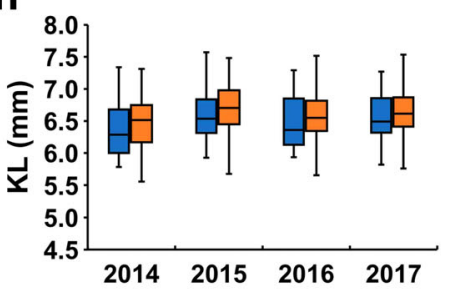

g
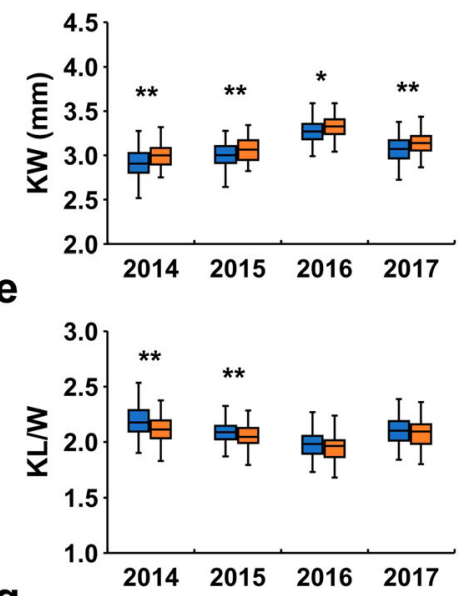
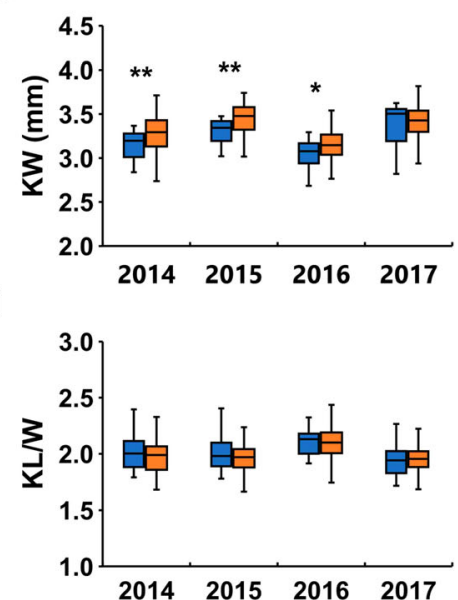

Fig. 4 Allelic analysis with kernel traits of TaFT-D1 in PG-RIL and the natural population. a Allelic segregation of KASP marker for TaFT-D1 alleles. Comparison analysis of TaFT-D1 alleles with the thousand kernel weight (TKW, b), kernel length $(K L, \mathbf{c})$, kernel width (KW, d) and kernel length/ width (KL/W, e) of PG-RIL in four environments. Comparison analysis of TaFT-D1 alleles with the TKW (f), KL (g), KW (h) and KL/W (i) of the natural population consisted of 141 cultivar/lines in four environments. ${ }^{* *} P<0.01$ and ${ }^{*} P<0.05$ (two-tailed $t$ test) indicates a significant difference to the two haplotypes

\section{Diagnostic marker and marker-assisted selection} Abundance of diagnostic markers in wheat enables breeders to create better combinations and select favorable cultivars to meet local breeding goals [62]. To date, numerous SNP loci related to kernel traits have been identified in wheat by high-throughput SNP chips combined with bi-parental populations [1, 34, 39]. In the present study, a KASP marker was developed to distinguish two alleles of TaFT-D1 and verified in PG-RIL and a natural population consisted of 141 cultivar/lines (Fig. 
a

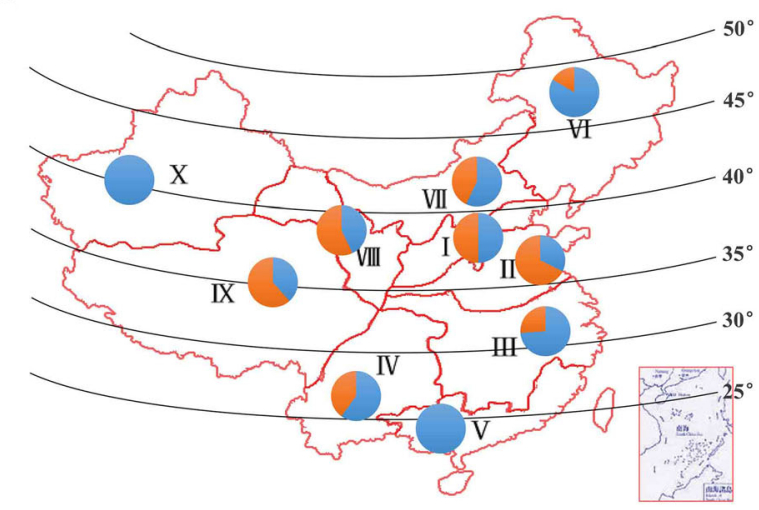

b

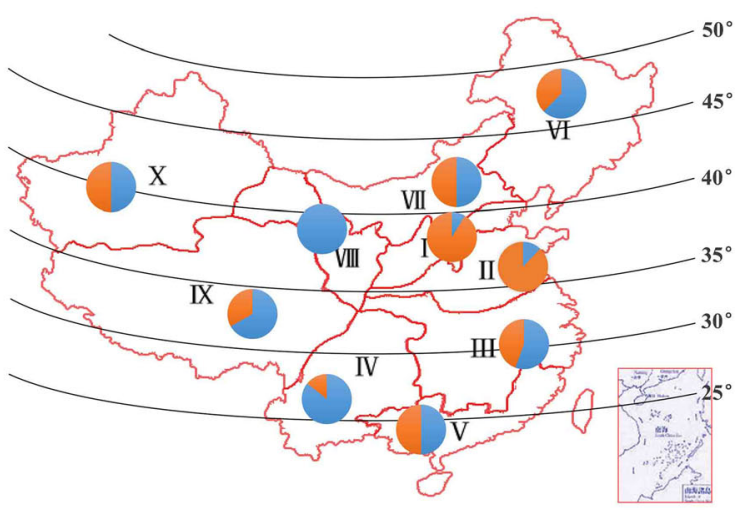

TaFT-D1(G) allele

Fig. 5 Geographic distribution of the TaFT-D1 alleles in the Chinese wheat ecological regions. Distribution of TaFT-D1 alleles in landraces (a) and modern cultivars (b) among ten Chinese ecological regions. I, northern winter wheat region; II, Yellow and Huai River valley winter wheat region; III, low and middle Yangtze River valley winter wheat region; IV, southwestern winter wheat region; V, southern winter wheat region; VI, northeastern spring wheat region; VII, northern spring wheat region; VIII, northwestern spring wheat region; IX, Qinghai-Tibet spring-winter wheat region; $X$, Xinjiang winter-spring wheat region

4). Furthermore, the alleles of TaFT-D1 were significantly associated with TKW and KW in both PG-RIL and natural populations (Fig. 4). G8901-allele, the favorable allele that produces higher TKW, was gradually accumulated during the wheat breeding process (Fig. 5). Therefore, the KASP marker can facilitate map-based cloning of QTKw.cas-7D.2 and QKw.cas-7D.1 and molecular-assisted selection breeding for high-yield in wheat.

\section{Conclusions}

In this study, we performed QTL analysis using the PGRIL population in four environments for kernel-related traits (TKW, KL, KW and KL/W), which were mainly distributed on chromosomes 1A, 1B, 4A, 5D, 6A, 7A and 7D (Fig. 3 and Additional file 2: Table S1). A total of 17 stable QTLs were identified in more than two individual environments (Table 3). Notably, the stable QTLs for TKW were mainly affected by KW (Table 4). Furthermore, the QTLs QTkw.cas-7D.2 and QKw.cas-7D.1 were delimited to the physical interval of approximately $3.82 \mathrm{Mb}$, and TaFT-D1 was considered as the candidate gene. Based on a $1 \mathrm{bp}$ InDel of TaFT-D1 between the two parents, a KASP marker of TaFT-D1 allele was developed and verified by PG-RIL and a natural population. The favorable TaFT-D1 (G)-allele associated with TKW and KW has been positively selected during Chinese wheat breeding. In addition, the current study provided new options for dissecting the genetic basis of yield and molecular-assisted breeding.

\section{Methods}

\section{Plant materials and field trials}

A mapping population composed of $176 \mathrm{~F}_{6-9}$ RILs derived from 'PuBing $3228 \times$ Gao 8901 'was developed by single seed descent method. The wheat germplasm P3228 was developed by Dr. Lihui Li (Chinese Academy of Agricultural Sciences). G8901 is a commercial cultivar released by Gaocheng institute of agricultural science, Hebei, China. The P3228 has higher kernel number per spike and the G8901 has higher thousand kernel weight (Fig. 1). A natural population consisted of 141 cultivar/ lines (maintained in our laboratory, Additional file 1: Table S7) was used for the KASP marker screening and two-tailed $\mathrm{t}$ test. The 176 RILs, with two parents and the natural population were grown at the Luancheng Agroecosystem Station, Chinese Academy of Sciences (37 $53^{\prime}$ $\left.15^{\prime \prime} \mathrm{N}, 114^{\circ} 40^{\prime} 47^{\prime \prime} \mathrm{E}\right)$ during four growing seasons from 2013 to 2014 to 2016-2017. In each environment, the RILs, two parents and the natural population were planted in a randomized complete block design with three replicates. A $1.5 \mathrm{~m}^{2}$ subplot with four $1.5 \mathrm{~m}$ long rows, $0.25 \mathrm{~m}$ apart, and 30 seeds for each row were used. The water, fertilizer and other management of all field trials were carried out in accordance with local standard practices. In addition, 150 landraces of the Chinese wheat mini-core collection [63] and 172 modern cultivars (maintained in our laboratory) were used to analyze the geographic distribution of TaFT-D1 alleles (Additional file 1: Table S8). The 150 landraces and 88 modern cultivars of the Chinese wheat mini-core collection were kindly provided by 
Dr. Xueyong Zhang (Chinese Academy of Agricultural Sciences).

\section{Phenotypic evaluation and statistical analysis}

For the four environments, 10 representative plants were sampled from each plot to investigate kernel-related traits. At seed maturity, the TKW and kernel morphometric traits (KL, KW and KL/W) of at least 500 kernels were measured three times using the rapid SC-G grain appearance quality image analysis system (WSeen Detection, Hangzhou, China). Analysis of variance (ANOVA), mean values of traits, standard deviations and variation coefficients $(\mathrm{CV})$ were performed with SPSS Statistics v20.0 software (SPSS, Chicago, USA). Effects among genotypes, environments, and GE interaction were estimated by ANOVA. BLUP for all four traits across four environments was calculated using $\mathrm{R}$ software (V.3.2.2; https://www.r-project.org/). The $H$ was calculated using the QGAStation 2.0 (http://ibi.zju.edu.cn/software/qga/ v2.0/index c.htm) and the following formula $H=V \mathrm{G} / V \mathrm{P}$; where $V G$ and $V \mathrm{P}$ are the genetic variance and phenotypic variance, respectively.

\section{QTL mapping}

The 'PuBing3228 $\times$ Gao 8901' RIL population and the two parents were genotyped by the Affymetrix wheat $660 \mathrm{~K}$ SNP array [64]. A total of 101,136 loci showed polymorphisms between P3228 and G8901. The linkage map comprised 23 linkage groups that consisted of 4477 bins, spanning $3529.5 \mathrm{cM}$ in length, with an average interval distance of $0.782 \mathrm{cM}$ between the adjacent markers. Linkage analysis was performed using JoinMap v4 [65], and the genetic map was drawn by Mapchart 2.0 [66]. The QTLs were scanned with QTL ICIMapping V4.1 [67] through inclusive composite interval mapping of additive and dominant QTL (ICIM-ADD) [67]. The LOD score to detect the presence of a QTL was above at 2.50 [68]. Digenic epistasis and environment interaction of QTLs were analyzed using QTL ICIMapping V4.1 through inclusive composite interval mapping of epistatic QTL (ICIM-EPI) [69]. The LOD score to detect the digenic epistasis QTL was above at 5.0 [68]. The QTL $\times$ environment interactions were scanned with QTL ICIMapping V4.1 through inclusive composite interval mapping of additive and dominant QTL (ICIM-ADD) [70]. QTLs with overlapping confidence intervals were regarded as the congruent QTLs. The QTLs were named based on McIntosh et al. [71], 'cas' represents Chinese Academy of Science.

Conditional QTL analysis was performed to analyze the genetic contributions of kernel-related traits to TKW, by the procedure of inclusive composite interval mapping [23]. The conditional phenotypic values $\left(y_{(\mathrm{TKW} \mid \mathrm{KL})}\right)$ of TKW in wheat were obtained by the mixed-model approach. The conditional phenotypic value can be partitioned as.
$y_{(\mathrm{TKW} \mid \mathrm{KL})=} \mu_{(\mathrm{TKW} \mid \mathrm{KL})}+G_{(\mathrm{TKW} \mid \mathrm{KL})}+E_{(\mathrm{TKW} \mid \mathrm{KL})}+e_{(\mathrm{TKW} \mid \mathrm{KL})}$. where $(\mathrm{TKW} \mid \mathrm{KL})$ denote TKW conditional on KL; $y_{(\mathrm{TKW} \mid \mathrm{KL})}$ is the conditional phenotypic value of TKW on $K L ; \mu_{(T K W \mid K L)}$ is the conditional population mean, $G_{(\mathrm{TKW} \mid \mathrm{KL})}$ is the conditional general genotypic effect; $E_{(\mathrm{TKW} \mid \mathrm{KL})}$ is the conditional effect for the environment and $e_{(\mathrm{TKW} \mid \mathrm{KL})}$ is the conditional residual error.

The conditional phenotypic values $y_{\text {(TKW } \mid \mathrm{KL})}, y_{(\mathrm{TKW} \mid \mathrm{KW})}$ and $\left.y_{(\mathrm{TKW} \mid \mathrm{KL} / \mathrm{W})}\right)$ are the conditional phenotypic value of TKW on KL, KW or KL/KW in the corresponding environment, which were estimated using QGAStation2.0 (http://ibi.zju.edu.cn/software/qga/). MapChart 2.2 (http:// www.biometris.nl/uk/Software/MapChart/) was used to draw the genetic map.

\section{Comparison of QTLs related to kernel traits}

We used flanking SNP markers sequence of QTLs to BLAST against the reference genome of Chinese Spring to acquire the physical position of the region [49]. High confidence candidate genes in the target interval were retrieved based on coding sequences (IWGSC_RefSeq_Annotations v1.0), and were further analyzed on NCBI Non-redundant protein sequences for function annotations. The expression profile database of nine candidate genes was blasted based on Chinese Spring cv-1 Development (pair) [50].

\section{Conversion of SNPs to KASP markers}

The SNPs tightly linked to two stable QTLs QTkw.cas7D.2 and QKw.cas-7D.1 and the $1 \mathrm{bp}$ InDel of TaFT-D1 were converted to KASP markers (Additional file 1: Table S9). KASP reactions were carried out on a BIORAD CFX real-time PCR system using the KASP V4.0 2× Mastermix (LGC Genomics, Teddington, UK) as previously described [1]. The fluorescence was monitored using Bio-Rad CFX Manage 3.1 software (LGC Genomics, Teddington, UK). Two-tailed $t$ test was performed with SPSS Statistics v20.0 software (SPSS, Chicago, USA).

\section{Supplementary information}

Supplementary information accompanies this paper at https://doi.org/10. 1186/s12870-020-02661-4.

Additional file 1 Table S1 Analysis of variance for the investigated traits of the PG-RIL in four environments. Table S2 Putative additive QTL associated with kernel related traits in four environments. Table S3 Epistatic effects and environmental interactions of QTLs for TKW, KL, KW and $\mathrm{KL} / \mathrm{W}$ in wheat detected from the PG-RIL population. Table S4 QTL $\times$ environment interactions for $\mathrm{TKW}, \mathrm{KL}, \mathrm{KW}$ and $\mathrm{KL} / \mathrm{W}$ in wheat detected from the PG-RIL population. Table S5 Unconditional and conditional QTLs for TKW in wheat. Table S6 Annotated genes harbored in the interval of QTKW.cas-7D.2 and QKW.cas-7D.1. Table S7 Detailed information on a natural population consisted of 141 cultivar/lines and their alleles of TaFT-D1. Table S8 Detailed information on 150 landraces and 172 modern cultivars and their alleles of TaFT-D1. Table S9 Primers used in this study.

Additional file 2 Fig. $\mathbf{S 1}$ Collinearity between the genetic (left) and physical (right) positions for SNPs mapped on the chromosome 7DS in PG-RIL genetic map. Fig. S2 A 1 bp InDel in TaFT-D1 caused a frameshift 
mutation of the protein. (a) Sequence alignment of TaFT-D1 showing 1 bp InDel between P3228 and G8901. (b) Protein alignment of TaFT-D1 showing frameshift mutation in P3228. Fig. $\mathbf{S 3}$ Heatmap showing the expression profile of DEGs at 15 development stages. Fig. S4 Allelic segregation of KASP markers AX-111061288 (a) and AX-111184541 (b) for QTKW.cas-7D.2 and QKW.cas-7D.1.

\section{Abbreviations}

BLUP: best linear unbiased predictors; ICIM: inclusive composite interval mapping; G8901: Gao8901; KASP: Kompetitive Allele-Specific PCR; KL: kernel length; KW: kernel width; LOD: threshold log-of-odds; P3228: PuBing 3228; PG-RIL: 'PuBing3228 × Gao 8901' recombinant inbred lines; PVE: phenotypic variance explained; QTL: quantitative trait loci; SNP: single nucleotide polymorphism; TKW: thousand kernel weight

\section{Acknowledgements}

The authors thank Dr. Xueyong Zhang providing the Chinese mini-core collection, including 157 landraces and 88 modern cultivars.

\section{Authors' contributions}

$\mathrm{LL}$ and DA conceived the study. $X Z, H L, F M$ and $Y C$ evaluated the phenotype. HL and XZ carried out QTL mapping, predicted candidate gene, and developed the KASP markers. JZ and YX constructed the RIL population. $\mathrm{HL}$ and $X Z$ analyzed data and wrote the manuscript. LL and DA supervised and revised the writing of the article. All authors approved the final manuscript.

\section{Funding}

This research was financially supported by the Strategic Priority Research Program of the Chinese Academy of Sciences (no. XDA24030102), the National Key Research and Development Program of China (no. 2016YFD0100102), and the National Natural Science Foundation of China (no. 31771787). The funding bodies were not involved in the design of the study, and collection, analysis, and interpretation of data, and manuscript writing.

\section{Availability of data and materials}

All the data generated or analyzed during the current study were included in the manuscript and its additional files. The raw data is available from the corresponding author on reasonable request. The collection of materials used in current study complied with institutional, national, or international guidelines.

\section{Ethics approval and consent to participate}

Not applicable.

\section{Consent for publication}

Not applicable.

\section{Competing interests}

The authors declare that they have no conflict of interest.

\section{Author details}

${ }^{1}$ Center for Agricultural Resources Research, Institute of Genetics and Developmental Biology, Chinese Academy of Sciences, Shijiazhuang 050021, China. ${ }^{2}$ The National Key Facility for Crop Gene Resources and Genetic Improvement, Institute of Crop Science, Chinese Academy of Agricultural Sciences, Beijing 100081, China. ${ }^{3}$ The Innovation Academy for Seed Design, Chinese Academy of Sciences, Beijing 100101, China. ${ }^{4}$ University of Chinese Academy of Sciences, Beijing 100049, China.

Received: 22 March 2020 Accepted: 23 September 2020

Published online: 23 November 2020

\section{References}

1. Ma F, Xu Y, Ma Z, Li L, An D. Genome-wide association and validation of key loci for yield-related traits in wheat founder parent Xiaoyan 6. Mo breeding. 2018;38(7):91.
2. Sehgal D, Mondal S, Guzman C, Barrios G, Franco C, Singh R, et al. Validation of candidate gene-based markers and identification of novel loci for thousand-grain weight in spring bread wheat. Front Plant Sci. 2019;10:3389.

3. Su Z, Jin S, Lu Y, Zhang G, Chao S, Bai G. Single nucleotide polymorphism tightly linked to a major QTL on chromosome 7A for both kernel length and kernel weight in wheat. Mol Breeding. 2016;36(2):15.

4. Würschum T, Leiser WL, Langer SM, Tucker MR, Longin CFH. Phenotypic and genetic analysis of spike and kernel characteristics in wheat reveals longterm genetic trends of grain yield components. Theor Appl Genet. 2018; 131(10):2071-84.

5. Fan C, Xing Y, Mao H, Lu T, Han B, Xu C, et al. GS3, a major QTL for grain length and weight and minor QTL for grain width and thickness in rice, encodes a putative transmembrane protein. Theor Appl Genet. 2006;112(6):1164-71.

6. Song X, Huang W, Shi M, Zhu M, Lin H. A QTL for rice grain width and weight encodes a previously unknown RING-type E3 ubiquitin ligase. Nat Genet. 2007;39(5):623-30.

7. Li Y, Fan C, Xing Y, Jiang Y, Luo L, Sun L, et al. Natural variation in GS5 plays an important role in regulating grain size and yield in rice. Nat Genet. 2011; 43(12):1266.

8. Wang S, Wu K, Yuan Q, Liu X, Liu Z, Lin X, et al. Control of grain size, shape and quality by OSSPL16 in rice. Nat Genet. 2012;44(8):950.

9. Zhang X, Wang J, Huang J, Lan H, Wang C, Yin C, et al. Rare allele of OsPPKL 1 associated with grain length causes extra-large grain and a significant yield increase in rice. Proc Natl Acad Sci U S A. 2012;109(52):21534-9.

10. Si L, Chen J, Huang X, Gong H, Luo J, Hou Q, et al. OsSPL13 controls grain size in cultivated rice. Nat Genet. 2016;48(4):447.

11. Liu J, Chen J, Zheng X, Wu F, Lin Q, Heng Y, et al. GW5 acts in the brassinosteroid signalling pathway to regulate grain width and weight in rice. Nat Plants. 2017:3(5):17043.

12. Wu W, Liu X, Wang M, Meyer R, Luo X, Ndjiondjop M, et al. A singlenucleotide polymorphism causes smaller grain size and loss of seed shattering during African rice domestication. Nat Plants. 2017;3(6):17064.

13. Simmonds J, Scott P, Brinton J, Mestre TC, Bush M, Del Blanco A, et al. A splice acceptor site mutation in TaGW2-A1 increases thousand grain weight in tetraploid and hexaploid wheat through wider and longer grains. Theor Appl Genet. 2016;129(6):1099-112.

14. Qin L, Hao C, Hou J, Wang Y, Li T, Wang L, et al. Homologous haplotypes, expression, genetic effects and geographic distribution of the wheat yield gene TaGW2. BMC Plant Biol. 2014;14(1):107.

15. Zhang $Y$, Li D, Zhang D, Zhao X, Cao X, Dong L, et al. Analysis of the functions of TaGW2 homoeologs in wheat grain weight and protein content traits. Plant J. 2018;94(5):857-66.

16. Liu H, Li H, Hao C, Wang K, Wang Y, Qin L, et al. TaDA1, a conserved negative regulator of kernel size, has an additive effect with TaGW2 in common wheat (Triticum aestivum L.). Plant Biotechnol J. 2020;18(5):1330-42.

17. Ma L, Li T, Hao C, Wang Y, Chen X, Zhang X. TaGS5-3A, a grain size gene selected during wheat improvement for larger kernel and yield. Plant Biotechnol J. 2016;14(5):1269-80.

18. Sajjad M, Ma X, Khan SH, Shoaib M, Song Y, Yang W, et al. TaFlo2-A1, an ortholog of rice Flo2, is associated with thousand grain weight in bread wheat (Triticum aestivum L.). BMC Plant Biol. 2017:17(1):164.

19. Jiang $Y$, Jiang Q, Hao C, Hou J, Wang L, Zhang H, et al. A yield-associated gene $\mathrm{TaCW}$, in wheat: its function, selection and evolution in global breeding revealed by haplotype analysis. Theor Appl Genet. 2014;128(1):131-43.

20. Hou J, Li T, Wang Y, Hao C, Liu H, Zhang X. ADP-glucose pyrophosphorylase genes, associated with kernel weight, underwent selection during wheat domestication and breeding. Plant Biotechnol J. 2017;15(12):1533-43.

21. Hou J, Jiang Q, Hao C, Wang Y, Zhang H, Zhang X. Global lection on sucrose synthase haplotypes during a century of wheat breeding. Plant Physiol. 2014;164(4):1918-29.

22. Zhu J. Analysis of conditional genetic effects and variance components in developmental genetics. Genetics. 1995;141:1633-9.

23. Li Q, Zhang Y, Liu T, Wang F, Liu K, Chen J, et al. Genetic analysis of kernel weight and kernel size in wheat (Triticum aestivum L.) using unconditional and conditional QTL mapping. Mol Breeding. 2015;35(10):194.

24. Zhang $X$, Deng Z, Wang Y, Li J, Tian J. Unconditional and conditional QTL analysis of kernel weight related traits in wheat (Triticum aestivum L.) in multiple genetic backgrounds. Genetica. 2014;142(4):371-9.

25. Ding A, Cui F, Li J, Zhao C, Wang L, Qi X, et al. QTL mapping for grain yield conditioned on its component traits in two RIL populations of bread wheat. Cereal Res Commun. 2013;41(1):45-53. 
26. Cui F, Li J, Ding A, Zhao C, Wang L, Wang X, et al. Conditional QTL mapping for plant height with respect to the length of the spike and internode in two mapping populations of wheat. Theor Appl Genet. 2011;122(8):1517-36.

27. Zhang N, Fan X, Cui F, Zhao C, Zhang W, Zhao X, et al. Characterization of the temporal and spatial expression of wheat (Triticum aestivum L.) plant height at the QTL level and their influence on yield-related traits. Theor Appl Genet. 2017;130(6):1235-52.

28. Xu Y, Wang R, Tong Y, Zhao H, Xie Q, Liu D, et al. Mapping QTLs for yield and nitrogen-related traits in wheat: influence of nitrogen and phosphorus fertilization on QTL expression. Theor Appl Genet. 2014;127(1):59-72.

29. Wang S, Wong D, Forrest K, Allen A, Chao S, Huang BE, et al. Characterization of polyploid wheat genomic diversity using a high-density 90,000 single nucleotide polymorphism array. Plant Biotechnol J. 2014;12(6): 787-96.

30. Winfield MO, Allen AM, Burridge AJ, Barker GL, Benbow HR, Wilkinson PA, et al. High-density SNP genotyping array for hexaploid wheat and its secondary and tertiary gene pool. Plant Biotechnol J. 2016;14(5):1195-206.

31. Zhou S, Zhang J, Che Y, Liu W, Lu Y, Yang X, et al. Construction of Agropyron Gaertn. Genetic linkage maps using a wheat 660K SNP array reveals a homoeologous relationship with the wheat genome. Plant Biotechnol J. 2018;16(3):818-27.

32. Cui F, Fan X, Chen M, Zhang N, Zhao C, Zhang W, et al. QTL detection for wheat kernel size and quality and the responses of these traits to low nitrogen stress. Theor Appl Genet. 2015;129(3):469-84.

33. Brinton J, Simmonds J, Minter F, Leverington-Waite M, Snape J, Uauy C. Increased pericarp cell length underlies a major quantitative trait locus for grain weight in hexaploid wheat. New Phytol. 2017;215(3):1026-38.

34. Ma J, Zhang H, Li S, Zou Y, Li T, Liu J, et al. Identification of quantitative trait loci for kernel traits in a wheat cultivar Chuannong16. BMC Genet. 2019;20:77.

35. Cheng X, Xin M, Xu R, Chen Z, Cai W, Chai L, et al. A single amino acid substitution in STKC_GSK3 kinase conferring semispherical grains and its implications for the origin of Triticum sphaerococcum Perc. Plant Cell. 2020; 32(4):923-34.

36. Wu Q, Chen Y, Zhou S, Fu L, Chen J, Xiao Y, et al. High-density genetic linkage map construction and QTL mapping of grain shape and size in the wheat population Yanda1817 × Beinong6. PLoS One. 2015;10(2):e0118144.

37. Cheng R, Kong Z, Zhang L, Xie Q, Jia H, Yu D, et al. Mapping QTLs controlling kernel dimensions in a wheat inter-varietal RIL mapping population. Theor Appl Genet. 2017;130(7):1405-14.

38. Su Q, Zhang X, Zhang W, Zhang N, Song L, Liu L, et al. QTL detection for kernel size and weight in bread wheat (Triticum aestivum L.) using a highdensity SNP and SSR-based linkage map. Front Plant Sci. 2018;9:1484.

39. Chen Z, Cheng X, Chai L, Wang Z, Bian R, Li J, et al. Dissection of genetic factors underlying grain size and fine mapping of QTgw.cau-7D in common wheat (Triticum aestivum L.). Theor Appl Genet. 2019;133:149-62.

40. Guan P, Di N, Mu Q, Shen X, Wang Y, Wang X, et al. Use of near-isogenic lines to precisely map and validate a major QTL for grain weight on chromosome 4AL in bread wheat (Triticum aestivum L.). Theor Appl Genet. 2019;132(8):2367-79.

41. Golan G, Ayalon I, Perry A, Zimran G, Ade-Ajayi T, Mosquna A, et al. GNI-AI mediates trade-off between grain number and grain weight in tetraploid wheat. Theor Appl Genet. 2019;1(8):2353-65.

42. Sakuma S, Golan G, Guo Z, Ogawa T, Tagiri A, Sugimoto K, et al. Unleashing floret fertility in wheat through the mutation of a homeobox gene. Proc Natl Acad Sci U S A. 2019;116(11):5182-7.

43. Mir RR, Kumar N, Jaiswal V, Girdharwal N, Prasad M, Balyan HS, et al. Genetic dissection of grain weight in bread wheat through quantitative trait locus interval and association mapping. Mol Breeding. 2012;29(4):963-72.

44. Guan P, Lu L, Jia L, Kabir MR, Zhang J, Lan T, et al. Global QTL analysis identifies genomic regions on chromosomes $4 \mathrm{~A}$ and $4 \mathrm{~B}$ harboring stable loci for yield-related traits across different environments in wheat (Triticum aestivum L.). Front Plant Sci. 2018;9:529.

45. Cui F, Zhao C, Ding A, Li J, Wang L, Li X, et al. Construction of an integrative linkage map and QTL mapping of grain yield-related traits using three related wheat RIL populations. Theor Appl Genet. 2014;127(3):659-75.

46. Zhai H, Feng Z, Du X, Song Y, Liu X, Qi Z, et al. A novel allele of TaGW2-A1 is located in a finely mapped QTL that increases grain weight but decreases grain number in wheat (Triticum aestivum L.). Theor Appl Genet. 2018;131(3): 539-53.

47. Gao F, Wen W, Liu J, Rasheed A, Yin G, Xia X, et al. Genome-wide linkage mapping of QTL for yield components, plant height and yield-related physiological traits in the Chinese wheat cross Zhou 8425B/Chinese spring. Front Plant Sci. 2015;6:1099.

48. Wang $X$, Dong L, Hu J, Pang $Y, H u$ L, Xiao G, et al. Dissecting genetic loci affecting grain morphological traits to improve grain weight via nested association mapping. Theor Appl Genet. 2019;132(11):3115-28.

49. International Wheat Genome Sequencing Consortium. Shifting the limits in wheat research and breeding using a fully annotated reference genome. Science. 2018;361(6403):eaar7191.

50. International Wheat Genome Sequencing Consortium. A chromosomebased draft sequence of the hexaploid bread wheat (Triticum aestivum) genome. Science. 2014;345(6194):1251788.

51. Zhuang QS. Chinese wheat improvement and pedigree analysis. Beijing: China Agricultural Press; 2003. p. 11.

52. Su Z, Hao C, Wang L, Dong Y, Zhang X. Identification and development of a functional marker of TaGW2 associated with grain weight in bread wheat (Triticum aestivum L.). Theor Appl Genet. 2011;122(1):211-23.

53. Röder MS, Huang $X Q$, Börner A. Fine mapping of the region on wheat chromosome 7D controlling grain weight. Funct Integr Genomic. 2008;8(1): 79-86.

54. Kamran A, lqbal M, Spaner D. Flowering time in wheat (Triticum aestivum L.): key factor for global adaptability. Euphytica. 2014;197(1):1-26.

55. Cao RF, Guo LJ, Ma M, Zhang WJ, Liu XL, Zhao HX. Identification and functional characterization of squamosa promoter binding protein-like gene TaSPL16 in wheat (Triticum aestivum L.). front. Plant Sci. 2019;10:212.

56. Ma L, Hao C, Liu H, Hou J, Li T, Zhang X. Diversity and sub-functionalization of TaGW8 homoeologs hold potential for genetic yield improvement in wheat. Crop J. 2019;7(6):830-44.

57. Liu H, Li T, Wang Y, Zheng J, Li H, Hao C, et al. TaZIM-A1 negatively regulates flowering time in common wheat (Triticum aestivum L.). J Integr Plant Biol. 2019;61(3):359-76.

58. Yan L, Fu D, Li C, Blechl A, Tranquilli G, Bonafede M, et al. The wheat and barley vernalization gene VRN3 is an orthologue of FT. Proc Natl Acad Sci U S A. 2006;103(51):19581-6.

59. Chen A, Li C, Hu W, Lau MY, Lin H, Rockwell NC, et al. PHYTOCHROME C plays a major role in the acceleration of wheat flowering under long-day photoperiod. Proc Natl Acad Sci U S A. 2014;111(28):10037-44.

60. Boden SA, Cavanagh C, Cullis BR, Ramm K, Greenwood J, Finnegan EJ, et al. $P p d-1$ is a key regulator of inflorescence architecture and paired spikelet development in wheat. Nat Plants. 2015;1(2):14016.

61. Liu H, Song S, Xing Y. Beyond heading time: FT-like genes and spike development in cereals. J Exp Bot. 2019;70(1):1-3.

62. Zheng J, Liu H, Wang Y, Wang L, Chang $X$, Jing R, et al. TEF-7A, a transcript elongation factor gene, influences yield-related traits in bread wheat (Triticum aestivum L.). J Exp Bot. 2014;65(18):5351-65.

63. Zhao J, Wang Z, Liu H, Zhao J, Tian L, Hou J, Zhang X, et al. Global status of 47 major wheat loci controlling yield, quality, adaptation and stress resistance selected over the last century. BMC Plant Biol. 2019;19(1):5.

64. Sun C, Dong Z, Zhao L, Ren Y, Zhang N, Chen F. The wheat 660K SNP array demonstrates great potential for marker-assisted selection in polyploid wheat. Plant Biotechnol J. 2020;18(6):1354-60.

65. Van Ooijen JW. JoinMap ${ }^{\oplus}$, software for the calculation of genetic linkage maps in experimental populations. Wageningen, Kyazma BV; 2020.

66. Voorrips RE. MapChart: software for the graphical presentation of linkage maps and QTLs. J Hered. 2002;93(1):77-8.

67. Meng L, Li H, Zhang L, Wang J. QTL IciMapping: integrated software for genetic linkage map construction and quantitative trait locus mapping in biparental populations. Crop J. 2015;3(3):269-83.

68. Sun Z, Li H, Zhang L, Wang J. Properties of the test statistic under null hypothesis and the calculation of LOD threshold in quantitative trait loci (QTL) mapping. Acta Agronomica Sinica. 2013;39:1-11.

69. Wang J. Inclusive composite interval mapping of quantitative trait genes. Acta Agronomica Sinica. 2009;35:239-45.

70. Li S, Wang J, Zhang L. Inclusive Composite Interval Mapping of QTL by Environment Interactions in Biparental Populations. PLOS ONE 2015;10(7): e0132414.

71. McIntosh RA, Dubcovsky J, Rogers WJ, Xia XC, Raupp WJ. Catalogue of gene symbols for wheat: 2018 supplement. Annu Wheat Newsl. 2018;64:73-93.

\section{Publisher's Note}

Springer Nature remains neutral with regard to jurisdictional claims in published maps and institutional affiliations. 